\title{
Probability of acoustic transmitter detections by receiver lines in Lake Huron: results of multi-year field tests and simulations
}

\author{
Todd A. Hayden ${ }^{\text {* }}$, Christopher M. Holbrook ${ }^{2}$, Thomas R. Binder ${ }^{1}$, John M. Dettmers ${ }^{3}$, Steven J. Cooke ${ }^{4}$ \\ Christopher S. Vandergoot ${ }^{5}$ and Charles C. Krueger ${ }^{6}$
}

\begin{abstract}
Background: Advances in acoustic telemetry technology have led to an improved understanding of the spatial ecology of many freshwater and marine fish species. Understanding the performance of acoustic receivers is necessary to distinguish between tagged fish that may have been present but not detected and from those fish that were absent from the area. In this study, two stationary acoustic transmitters were deployed $250 \mathrm{~m}$ apart within each of four acoustic receiver lines each containing at least 10 receivers (i.e., eight acoustic transmitters) located in Saginaw Bay and central Lake Huron for nearly 2 years to determine whether the probability of detecting an acoustic transmission varied as a function of time (i.e., season), location, and distance between acoustic transmitter and receiver. Distances between acoustic transmitters and receivers ranged from $200 \mathrm{~m}$ to $>10 \mathrm{~km}$ in each line. The daily observed probability of detecting an acoustic transmission was used in simulation models to estimate the probability of detecting a moving acoustic transmitter on a line of receivers.
\end{abstract}

Results: The probability of detecting an acoustic transmitter on a receiver $1000 \mathrm{~m}$ away differed by month for different receiver lines in Lake Huron and Saginaw Bay but was similar for paired acoustic transmitters deployed $250 \mathrm{~m}$ apart within the same line. Mean probability of detecting an acoustic transmitter at $1000 \mathrm{~m}$ calculated over the study period varied among acoustic transmitters $250 \mathrm{~m}$ apart within a line and differed among receiver lines in Lake Huron and Saginaw Bay. The simulated probability of detecting a moving acoustic transmitter on a receiver line was characterized by short periods of time with decreased detection. Although increased receiver spacing and higher fish movement rates decreased simulated detection probability, the location of the simulated receiver line in Lake Huron had the strongest effect on simulated detection probability.

Conclusions: Performance of receiver lines in Lake Huron varied across a range of spatiotemporal scales and was inconsistent among receiver lines. Our simulations indicated that if $69 \mathrm{kHz}$ acoustic transmitters operating at $158 \mathrm{~dB}$ in 10-30 $\mathrm{m}$ of freshwater were being used, then receivers should be placed $1000 \mathrm{~m}$ apart to ensure that all fish moving at $1 \mathrm{~m} \mathrm{~s}^{-1}$ or less will be detected $90 \%$ of days over a 2 -year period. Whereas these results can be used as general guidelines for designing new studies, the irregular variation in acoustic transmitter detection probabilities we observed among receiver line locations in Lake Huron makes designing receiver lines in similar systems challenging and emphasizes the need to conduct post hoc analyses of acoustic transmitter detection probabilities.

Keywords: Detection probability, Simulation, Great Lakes, Range testing, Acoustic telemetry, Generalized additive models

\footnotetext{
*Correspondence: thayden@usgs.gov

${ }^{1}$ Center for Systems Integration and Sustainability, Department

of Fisheries and Wildlife, Michigan State University, Hammond Bay

Biological Station, 11188 Ray Road, Millersburg, MI 49759, USA

Full list of author information is available at the end of the article
} 


\section{Background}

Animal movement is a fundamental process for structuring populations and communities, and determining the fate of individuals $[1,2]$. In aquatic systems, fishes move across a wide range of spatial and temporal scales to obtain transitory resources, avoid predators, and respond to physical constraints imposed by the environment [3, 4]. Recent advances in acoustic telemetry technology and methodology have improved understanding of fish movements at spatial and temporal scales that were previously not possible and have provided insights into fish habitat use, spawning, site fidelity, and migration [5-8]. Furthermore, advancements in animal biologging and telemetry technology have provided insight into development and structure of social interactions of many terrestrial animals [9].

The quality of information obtained from a telemetry system in terrestrial or aquatic systems depends on many biotic and abiotic aspects of the transmitter-receiver system $[9,10]$. In an aquatic system, an acoustic transmitter propagates a signal through water that is detected, decoded, and recorded by an acoustic receiver (and hydrophone) (herein referred to as a receiver) [7]. The distance between an acoustic transmitter and receiver in which an acoustic transmission can be detected depends on abiotic and biotic variables that influence the rate of energy attenuation as the signal passes through the water and on sources of noise that interfere with the receiver's ability to identify and decode the signal. Variables that influence the strength of a signal when it reaches a receiver include acoustic transmitter output power, spreading losses, signal refraction, receiver orientation, turbidity, air entrainment, water column stratification, algal blooms, ice cover, and submerged vegetation [7, 11-17]. The probability of acoustic transmitter detection is partly a function of the signal-to-noise ratio; noises that can interfere with signal detection may be generated by water flowing over substrate or mooring equipment (including the receiver), precipitation on the water surface, waves, grinding ice, anthropogenic sources (e.g., boats, sonars, pumps, vehicles), or other ambient biological noise (e.g., shrimp) [7]. In addition to abiotic and biotic variables that can influence the probability of acoustic transmitter detection, collision of signals from multiple acoustic transmitters arriving at the receiver at nearly the same time may prevent an acoustic transmitter from being decoded or result in an acoustic transmitter that is recorded incorrectly [18]. Numerous variables can affect detection range in a heterogeneous environment, resulting in variable detection ranges and probabilities at a receiver location over time.

Performance of acoustic telemetry receivers needs to be assessed to accurately describe fish behavior and reduce bias from unrecognized receiver performance problems [14]. Although acceptable performance of an acoustic system is project specific, poor or variable performance of an acoustic receiver line may result in periods of time when it is difficult to distinguish when a fish is present and not detected as compared to a fish that is absent from the area. Detection range is often evaluated before a telemetry study begins so as to inform the study design to be used, although retrospective analyses of detection range also have been used to evaluate performance [14]. Acoustic transmitter detection range represents the probability of detecting individual acoustic transmissions as a function of the distance between transmitter and receiver [19]. Static range testing uses fixed-position acoustic transmitters and receivers to estimate the proportion of acoustic transmissions detected over time and under varying environmental conditions [20]. Performance of receivers given existing environmental conditions and transmission characteristics of acoustic transmitters needs to be evaluated at temporal scales consistent with study objectives to fully quantify the effects of varying environmental conditions. Mobile range testing can also be used to evaluate receiver performance by 'swimming' an acoustic transmitter near a receiver or network of receivers to evaluate transmitter detection ranges as a function of environmental conditions and distance between the transmitter and receivers.

Receiver lines consisting of multiple receivers with overlapping detection ranges are commonly used to identify fish movements into or out of a specific area, or to observe fish movements along migratory pathways [21, 22]. Notwithstanding the effects of variables that cause attenuation or interference, the probability of detecting a moving fish on a stationary receiver line (i.e., acoustic transmitter detection probability of a line) depends on transmission rate, receiver spacing, fish movement rate, and acoustic transmitter detection range. Among these variables, acoustic transmission rate and receiver spacing are controlled by the researcher, but transmitter detection range and fish movement rates are not directly controlled and can vary unpredictably over time [17]. Rapid movements or low acoustic transmitter detection range may result in some individuals passing a receiver line without being detected [23]. The probability of detecting a moving fish by a stationary line of receivers may be estimated using mobile range testing and simulation or modeling $[23,24]$. Although range testing can provide insight into the performance of a single receiver, it can be difficult to predict or infer the probability of detecting a tagged fish moving through a line of stationary receivers.

Our objectives were (1) to characterize seasonal patterns in the probability of detecting an acoustic transmission at $1000 \mathrm{~m}$ at four receiver lines in Lake Huron, (2) to determine whether mean probability of detecting 
an acoustic transmission at a distance of $1000 \mathrm{~m}$ differed among receiver lines, and (3) to determine using simulation whether fish movement rates, receiver spacing, and the probability of detecting an acoustic transmission influenced the probability of detecting a tagged fish moving through a line of receivers. Objectives were addressed using static range testing at four receiver lines, each containing at least 10 receivers, in Lake Huron over 2 years. Two acoustic transmitters, located $250 \mathrm{~m}$ apart, were deployed within each receiver line for nearly 2 years. Distances between acoustic transmitters and receivers ranged from $200 \mathrm{~m}$ to $>10 \mathrm{~km}$ in each line. Logistic regression and generalized additive models (GAMs) were used to estimate weekly detection range curves for each acoustic transmitter, predict acoustic transmitter detection probability at $1000-\mathrm{m}$ transmitter-to-receiver distance, and estimate seasonal patterns in acoustic transmitter detection probability at $1000 \mathrm{~m}$. Simulation models were used to estimate the probability of detecting a fish moving through a line of acoustic receivers for different movement rates and receiver spacing scenarios. Simulation models were based on daily empirical detection range curves derived from one of each paired acoustic transmitters deployed in Lake
Huron and represented each of the four receiver lines in Lake Huron used in the field portion of the study. This paper represents part of a larger project investigating the movement patterns of walleye Sander vitreus in Lake Huron [24].

\section{Methods}

Acoustic transmitters (Vemco V16-4H, $158 \mathrm{~dB}$ re $1 \mu \mathrm{Pa}$ at $1 \mathrm{~m}$ output) were deployed in 2010 and 2011 within four receiver lines in Saginaw Bay and central Lake Huron (Fig. 1). At each receiver line, two acoustic transmitters were deployed approximately $250 \mathrm{~m}$ apart for at least 488 days (Table 1). Acoustic transmitters deployed within lines 1 and 2 were located in Saginaw Bay in approximately $13 \mathrm{~m}$ of water and transmitters deployed in lines 3 and 4 were located in Lake Huron in water ranging from 7 to $17 \mathrm{~m}$ in depth (Table 1). Two acoustic transmitters were deployed between two receivers such that the minimum distance between a transmitter and receiver was $200 \mathrm{~m}$. A 2-m stainless steel mooring cable attached to a concrete anchor and buoy was used to suspend the acoustic transmitter about $1 \mathrm{~m}$ above the lake bottom. Transmitters were deployed $1 \mathrm{~m}$ above the lake bottom because we assumed that most walleye would orient near

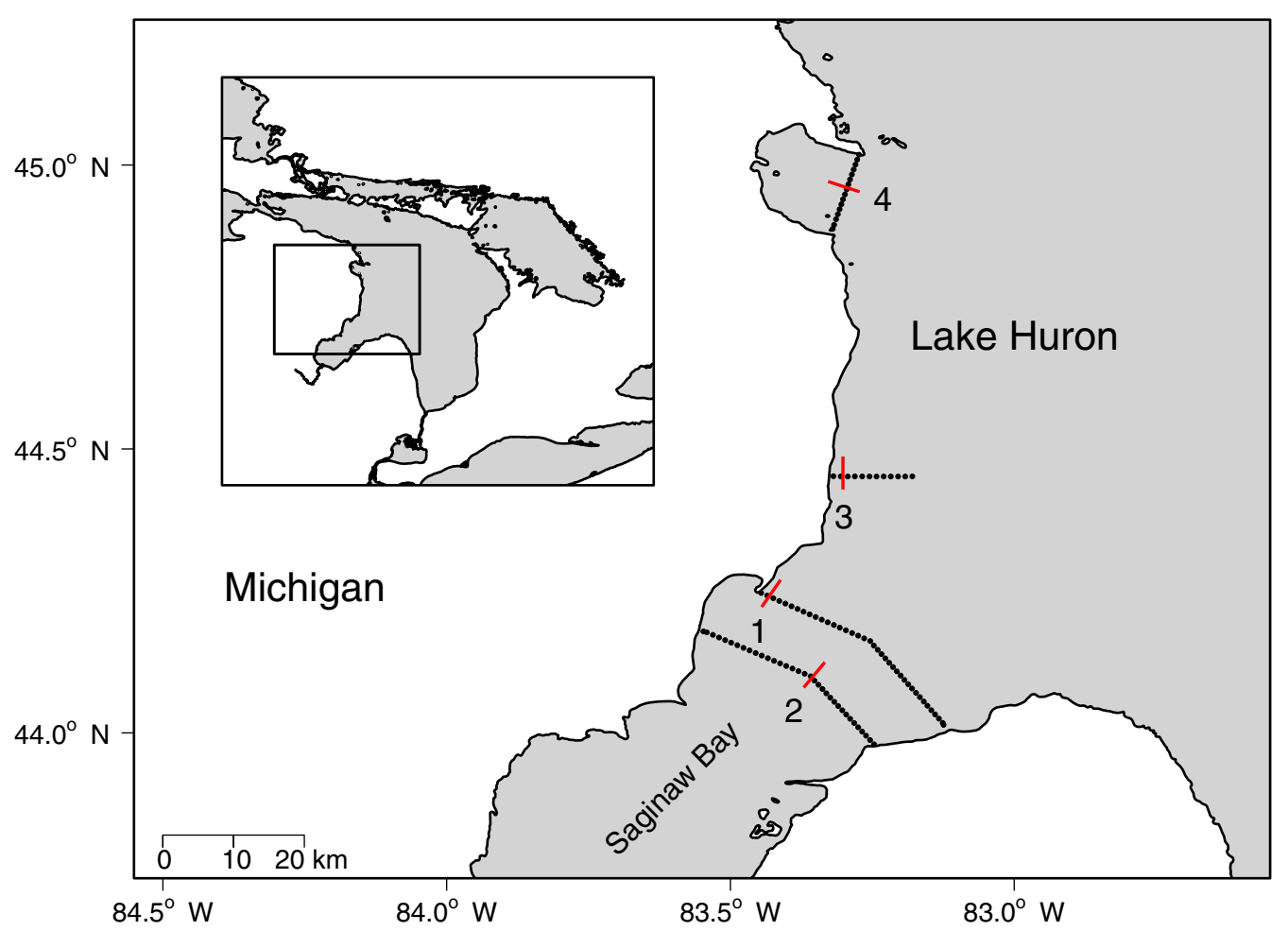

Fig. 1 Locations of acoustic transmitters and receiver lines in Saginaw Bay and Lake Huron in 2010-2012. Red lines are acoustic transmitters and small black circles are receivers. Two transmitters were deployed $250 \mathrm{~m}$ apart within each receiver line. Transmitters 1-2 were deployed in line 1, 3-4 at line 2, 5-6 at line 3, and 7-8 at line 4. Inset is Lake Huron 
Table 1 Summary of range testing at 4 acoustic receiver lines in Saginaw Bay and Lake Huron

\begin{tabular}{lllllllll}
\hline Tag & Line & Start & End & Depth $(\mathbf{m})$ & $\begin{array}{l}\text { Duration } \\
\text { (days) }\end{array}$ & Total detections & $\begin{array}{l}\text { Detections } \\
\text { day }\end{array}$ & $\begin{array}{c}\text { Max distance } \\
\text { detected (km) }\end{array}$ \\
\hline 1 & 1 & $2010-11-17$ & $2012-10-01$ & 13 & 684 & 358,669 & 524 & 11.8 \\
2 & 1 & $2010-11-17$ & $2012-10-01$ & 13 & 684 & 353,712 & 517 & 11.6 \\
3 & 2 & $2010-11-17$ & $2012-09-01$ & 14 & 654 & 204,032 & 312 & 10.8 \\
4 & 2 & $2010-11-17$ & $2012-09-01$ & 14 & 654 & 262,871 & 402 & 11.4 \\
5 & 3 & $2011-06-01$ & $2012-11-15$ & 7 & 533 & 132,032 & 248 & 9.8 \\
6 & 3 & $2011-06-01$ & $2012-11-15$ & 7 & 533 & 153,376 & 288 & 7.6 \\
7 & 4 & $2011-06-01$ & $2012-10-01$ & 17 & 488 & 212,519 & 435 & 6.8 \\
8 & 4 & $2011-06-01$ & $2012-10-01$ & 17 & 488 & 228,106 & 467 & 6.6 \\
\hline
\end{tabular}

Each line included two stationary acoustic transmitters (Tag) located approximately $250 \mathrm{~m}$ apart. 'Start' and 'end' is start and end dates of range testing trial, depth is approximate water depth (meters) at stationary transmitters, and duration is length of experimental trial (days). Dates are yyyy-mm-dd format. The total number of detections (tot. detections) was calculated by summing the number of detections on all receivers at each station during the trial. Average number of detections per day (detections day ${ }^{-1}$ ) was calculated by dividing the number of detections recorded for the trial by the duration of the experimental trial. The maximum distance an acoustic transmitter was detected at each site (max distance detected) is reported for each transmitter. See Fig. 1 for locations of stations

the lake bottom. Vertical position of transmitter in the water column can influence detection probability, but we did not evaluate position of walleye in the water column or the effects of vertical transmitter position on detection probability in this study. Acoustic transmitters were attached to the mooring cable using an 'L'-shaped wire hanger assembly to offset the transmitter from the mooring cable by approximately $12.5 \mathrm{~cm}$. The wire hanger was used to decrease interference of mooring cables on acoustic signals. Acoustic transmitters were programmed to emit coded acoustic bursts $(69 \mathrm{kHz})$ at random intervals between 300 and $900 \mathrm{~s}$ (mean interval $=600 \mathrm{~s}$ ).

Acoustic transmitters were detected on receivers deployed as part of the Great Lakes Acoustic Telemetry Observation System receiver network in Saginaw Bay and Lake Huron [24]. In 2010-2011, the acoustic receiver (VR2W, $69 \mathrm{kHz}$, omnidirectional, Vemco, Halifax, NS) network consisted of 15 lines of receivers deployed in Lake Huron with receivers placed at 1000-m intervals along a line. Acoustic receivers were attached to steel cables and suspended between concrete anchors and buoys in waters ranging from 2 to $35 \mathrm{~m}$. All receivers were retrieved annually for data download and battery replacement. Receivers in water depths of $<10 \mathrm{~m}$ were retrieved during winter 2010 and 2011 to prevent loss from ice flows and replaced in the springtime. Receivers in waters $>10 \mathrm{~m}$ were retrieved, downloaded, and immediately replaced with new receivers. Biofouling of recovered receivers was minimal and consisted of a thin biological film. Colonization of the external surface of the receiver by dreissenid mussel (Dreissena spp.) species common in the Great Lakes was minimal.

Detection data from all receivers were screened for false detections using a short-interval criterion [18]. The short-interval criterion assumed that more than one false detection of the same code within a short period of time was rare. Single detections for an acoustic transmitter within a 5 -h time interval on each receiver were identified as possible false detections and removed prior to subsequent analysis. Ninety-nine percent of all detections passed the filter.

Detection range curves for each acoustic transmitter, meaning the probability of detecting a single coded signal as a function of distance from the transmitter, were estimated using binomial-response logistic regression for each week of the experimental trial ( $>69$ weeks) [19]. The number of successful detections for each acoustic transmitter within a weekly time interval was calculated for all receivers that detected the transmitter at least once during the study. The number of missed detections for each transmitter and receiver was calculated by subtracting the number of successful acoustic transmitter detections from the expected number (calculated from average acoustic transmitter delay) of acoustic transmissions. Distances from the acoustic transmitter to all receivers that detected at least one transmission during the study were calculated from geographic coordinates of the acoustic transmitter and receivers and ranged from $202 \mathrm{~m}$ to $11.8 \mathrm{~km}$. Firth's bias-reduced logistic regression algorithm, as implemented in the R package 'brglm,' was used to estimate model coefficients for all range detection curves [25]. Firth's bias-reduced algorithm was used because acoustic transmissions detected on few $(<3)$ receivers in close proximity to the transmitter, and few or no transmissions detected on other receivers farther away, resulted in infinite maximum likelihood estimates using traditional maximum likelihood logistic regression [26]. Goodness of fit for logistic regression models was assessed using McFadden's pseudo $R^{2}$ [27]. McFadden's $R^{2}$ ranges from 0 to 1 with values close to 1 representing 
substantial improvement in the fit of the full model compared to the intercept-only model. Values of McFadden's pseudo $R^{2}$ are smaller than analogous $R^{2}$ used for linear regression, and pseudo $R^{2}$ values ranging from $0.2-0.4$ and are equivalent to $R^{2}$ values of $0.4-0.8$ in linear regression [28]. Model coefficients were used to estimate acoustic transmitter detection probability at $1000 \mathrm{~m}$ distance for each transmitter and weekly time interval (hereafter $D P_{1000}$ ). GAMs were used to describe nonlinear trends in time series of $D P_{1000}$ as a function of month, acoustic transmitter, and receiver line. GAMs are functionally related to generalized linear models but use nonparametric smoothing functions to describe nonlinear trends between predictor and response variables $[29,30]$. The 'gamm' function in the R package 'mgcv' fits additive models using regression spline smoothers and allows incorporation of autocorrelation, variance, and error structures. In this study, the 'gamm' function was used to fit models, and autocorrelation or variance structures were included in models when residual plots or autocorrelation plots indicated that the model did not account for heterogeneity or significant autocorrelation structure [31]. A cyclic spline accounts for the cyclic relationship between levels of a categorical predictor and was used to model the nonlinear trend between acoustic transmitter detection probability and month (i.e., months 1 and 12 are consecutive) [31].

Akaike information criterion (AIC) model selection was used to identify the best additive model, as defined by the smallest AIC score, from a suite of candidate models constructed from month, acoustic transmitter, and receiver line predictor variables to explain nonlinear trends in transmitter detection probability [32]. Candidate models were selected to determine whether seasonal patterns at the monthly time scale were similar for all acoustic transmitters, different among receiver lines (i.e., were similar between paired transmitters), or different for all transmitters. Conceptually, these hypotheses were tested by comparing explanatory power of candidate models that included a single nonlinear smoothing function fit to the acoustic transmission detection probability versus month relationship for all transmitters combined, separate nonlinear smoothing functions $(n=4)$ for each line, and separate nonlinear smoothing functions for each transmitter $(n=8)$.

In addition to modeling nonlinear trends in $D P_{1000}$ as a function of month, terms for 'transmitter' and 'line' were included in candidate models to estimate mean $D P_{1000}$ for each acoustic transmitter or line. Comparison of the explanatory power of candidate models that included or excluded these terms was used to determine whether mean $D P_{1000}$ differed among individual transmitters or receiver lines. Post hoc comparisons using the 'glht' function in the R package 'multcomp' were used to simultaneously test for differences among predicted mean $D P_{1000}$ for 'transmitter' or 'line' model terms [33]. Acoustic transmitter detection probability is bounded by 0 and 1 and may not be normally distributed; therefore, all data were $\operatorname{logit}$-transformed $[\log (x / 1-x)]$ before model fitting. The delta method was used to approximate the standard error of back-transformed $D P_{1000}$ for visualization [34]. Our analysis of $D P_{1000}$ followed a twostage approach that used logistic regression to estimate the range detection curve at weekly time intervals. These results were then incorporated into GAMs to characterize seasonal patterns in $D P_{1000}$. The two-stage approach did not explicitly accommodate within-week variability in detection probability as a function of distance but did allow us to account for autocorrelation structure in the data using GAMs.

Simulation models were constructed to estimate the probability of detecting a passing acoustic transmitter moving at a constant rate on one or more stationary receivers in a line (hereafter $D P_{\text {pass }}$ ) and were independent from analyses of $D P_{1000}$ described above (Additional file 1). Input variables for each simulation model included an empirically derived range detection curve (representing daily detection probability for a receiver-transmitter pair), a vector of inter-receiver distances, acoustic transmission frequency, and a constant fish movement rate (Additional file 1). The probability of detecting an acoustic transmission as a function of the distance between the acoustic transmitter and receiver (i.e., detection range curve) was estimated empirically by calculating the number of successful detections of each transmitter on all receivers that detected the transmitter at least once during each day of the study. The number of missed detections for each acoustic transmitter and receiver was calculated by subtracting the number of successful acoustic transmitter detections from the expected number of transmitter transmissions (calculated from average transmitter delay). The probability of detecting an acoustic transmission for each receiver was calculated by dividing the expected number of transmissions by the number of successful detections for each receiver. Empirical detection range curves for each day and acoustic transmitter were estimated by linear interpolation of detection probability calculated at each receiver as a function of distance. Empirical detection range curves were calculated for one acoustic transmitter within each line (acoustic transmitters 1,3,5,7) in Saginaw Bay and Lake Huron. Our use of empirical range detection curves in simulations did not allow us to estimate uncertainty in detection range within days. However, we used separate empirical range detection curves for each day of the study such that the full extent of variability in detection range among 
days was incorporated in season-wide simulation results. Multiple fish movement rates and receiver spacing were simulated to explore sensitivity of $D P_{\text {pass }}$ to receiver spacing and fish movement rates for daily range detection curves observed calculated for each acoustic transmitter. For each day, $D P_{\text {pass }}$ was simulated for three interreceiver distances $(500,1000$, or $3000 \mathrm{~m})$ and two fish movement rates $\left(0.5\right.$ or $\left.1.0 \mathrm{~m} \mathrm{~s}^{-1}\right)$. Thus, we evaluated six receiver spacing and movement rate scenarios for each daily range detection curve. All virtual receiver lines consisted of 10 equally spaced receivers. The 1000-m interreceiver distance was chosen to mimic receiver spacing of acoustic receivers deployed in a study of walleye movements in Lake Huron [24]; 500- and 3000-m interreceiver distances were arbitrarily chosen to encompass conceivable other receiver deployment scenarios. Swimming speeds of fish were chosen to represent the range of swimming speeds observed in walleye [35]. The starting location for each simulated fish was randomly chosen but constrained to $1000 \mathrm{~m}$ from the virtual receiver line, and a distance equal to half of the spacing past receivers at both ends of the simulated line and movement of virtual fish was perpendicular to receiver line. The perpendicular arrangement between the simulated receiver line and acoustic transmitter provided conservative estimates of $D P_{\text {pass }}$. Non-perpendicular paths provided additional time to detect virtual fish. Simulated acoustic transmission frequency mimicked transmitters implanted in walleye to study movements in Lake Huron and was the same for all simulations [24]. The time period between coded acoustic transmissions was random with a mean of $120 \mathrm{~s}$ and ranged from 60 to $180 \mathrm{~s}$. Detection or non-detection of each simulated acoustic transmission on each virtual receiver was determined by a draw from a Bernoulli distribution, where the probability of detection (success) was set from the daily detection range curve (from Lake Huron) and the transmitter-to-receiver distance. We also removed single detections of each virtual fish on each receiver to be consistent with the short-interval filter described above. $D P_{\text {pass }}$ for each day of the study in Lake Huron was calculated for each simulation scenario as the proportion of virtual fish detected out of 1000 simulated fish at each receiver line.

\section{Results}

More than 1.9 million acoustic transmissions were detected from eight stationary acoustic transmitters on 69 acoustic receivers set within four lines in Saginaw Bay and Lake Huron from November 2010 to November 2012 (Fig. 1; Table 1). The expected number of transmissions was 144 for each acoustic transmitter on each day. The average number of detections per day was similar between paired acoustic transmitters within receiver lines and ranged from approximately 250 to 500 for all transmitters (Table 1). The expected number of acoustic transmissions emitted ranged from 70,272 to 98,496 for all acoustic transmitters during the experiment. The highest number of detections per day occurred from acoustic transmitters 1 and 2 at line 1 and the maximum distance at which a transmission was detected varied and ranged from $7 \mathrm{~km}$ to more than $11 \mathrm{~km}$ (Table 1). Minimum transmitter-receiver distances varied among acoustic transmitters and ranged from approximately 200 to $400 \mathrm{~m}$. At all lines, detections at maximum detection range (7 to $>11 \mathrm{~km})$ occurred infrequently ( $<\sim 5$ times/line) during the 2-year period. Thus, the detection probability at these maximum observed detection ranges was nearly zero.

Detection rate by receivers in Lake Huron was inversely related to transmitter-receiver distance for all time intervals and all acoustic transmitters. The median decrease in detection rate for a $1-\mathrm{m}$ increase in distance between acoustic transmitters and receiver ranged from $0.03 \%$ to $0.67 \%$ (median: $0.12 \%$ ) for all 678 logistic regression models fit to weekly subsets of detections for each acoustic transmitter. All model coefficients differed from zero $(p<0.05)$. Model fit estimated by McFadden's pseudo$R^{2}$ ranged from 0.11 to 0.99 and was higher than 0.8 for $87 \%$ of models. Only 27 models had McFadden's pseudo$R^{2}$ values equal to or less than 0.6. Detection rate estimated for transmitter-receiver distances less than $400 \mathrm{~m}$ was lower than expected for some weekly intervals and acoustic transmitter locations.

Estimated values of detection probability at $1000 \mathrm{~m}$ $\left(D P_{1000}\right)$ fluctuated through time and ranged from less than 0.2 to 0.9 for all acoustic transmitters (Fig. 2). Patterns of variability in $D P_{1000}$ time series for paired acoustic transmitters within each line were more similar than patterns among lines (Fig. 2). The specific timing of high and low detection probability varied among lines and was characterized by sudden changes in detection probability spanning 1-2 weeks when compared to the overall mean detection probability at each line (Fig. 2).

The best model, as determined using AIC, included four nonlinear smoothing functions that represented the relationship between $D P_{1000}$ and month for combined acoustic transmitters at each line (Table 2). The $\triangle \mathrm{AIC}$ value calculated for this model and the next best candidate model was 7.8 (Table 2). A $\triangle$ AIC greater than six is considered strong support for one model over the other and likely reflects a true difference between models [32]. Comparison of Akaike weights for the two best models indicated that the top-ranked model was at least 49 times more strongly supported by the data than the second-ranked model. Strong support of the top-ranked model over candidate models that included smoothed terms for each acoustic transmitter or a single smoother 

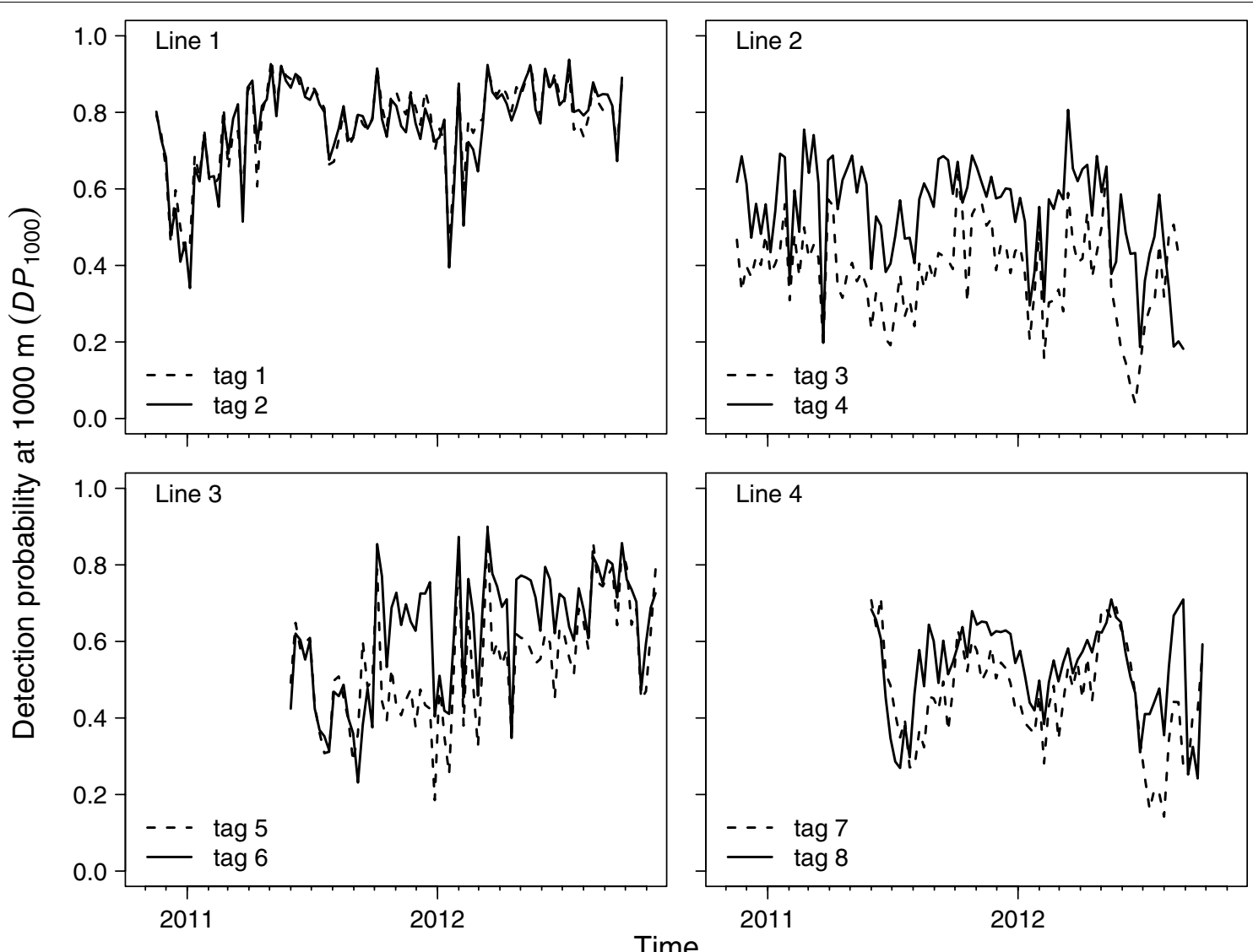

Fig. 2 Detection probability time series estimated for $1000 \mathrm{~m}$ transmitter-receiver distance $\left(D P_{1000}\right)$ in Lake Huron. $D P_{1000}$ was estimated for weekly intervals using logistic regression. Dashed and solid lines represent paired acoustic transmitters within each receiver line. Two transmitters (tag) were moored approximately $250 \mathrm{~m}$ apart at each line (panes). See Fig. 1 for locations

Table 2 Summary information for candidate models summarizing the probability of detecting a transmitter at $1000 \mathrm{~m}$ as a function of month and transmitter location (receiver line or transmitter)

\begin{tabular}{|c|c|c|c|c|c|c|}
\hline Candidate model & $d f$ & LL & AIC & $\Delta \mathrm{AIC}$ & Akaike weight & $R^{2}$ \\
\hline$D P_{1000_{i s}}=\alpha+f_{\text {line }}\left(\right.$ month $\left._{s}\right)+\operatorname{tag}_{i}+\varepsilon_{i s}$ & 14 & -526.74 & 1081.49 & 0.0 & 0.980 & 0.63 \\
\hline$D P_{1000_{i s}}=\alpha+f\left(\right.$ month $\left._{s}\right)+\operatorname{tag}_{i}+\varepsilon_{i s}$ & 11 & -533.66 & 1089.32 & 7.8 & 0.020 & 0.57 \\
\hline$D P_{1000_{i s}}=\alpha+f_{\text {tag }}\left(\right.$ month $\left._{s}\right)+\operatorname{tag}_{i}+\varepsilon_{i s}$ & 18 & -530.36 & 1096.73 & 15.24 & 0.000 & 0.59 \\
\hline$D P_{1000_{i s}}=\alpha+f_{\text {line }}\left(\right.$ month $\left._{s}\right)+$ line $+\varepsilon_{\text {is }}$ & 10 & -541.55 & 1103.11 & 21.62 & 0.000 & 0.54 \\
\hline$D P_{1000_{i s}}=\alpha+f\left(\right.$ month $\left._{s}\right)+$ line $+\varepsilon_{i s}$ & 7 & -547.85 & 1109.71 & 28.22 & 0.000 & 0.51 \\
\hline$D P_{1000_{i s}}=\alpha+f_{\text {tag }}\left(\right.$ month $\left._{s}\right)+$ line $+\varepsilon_{i s}$ & 14 & -543.79 & 1115.57 & 34.08 & 0.000 & 0.53 \\
\hline$D P_{1000_{i s}}=\alpha+f_{\text {line }}\left(\right.$ month $\left._{s}\right)+\varepsilon_{i s}$ & 7 & -590.51 & 1195.02 & 113.53 & 0.000 & 0.01 \\
\hline$D P_{1000_{i s}}=\alpha+f\left(\right.$ month $\left._{s}\right)+\varepsilon_{i s}$ & 4 & -596.57 & 1201.14 & 119.66 & 0.000 & 0.00 \\
\hline$D P_{1000_{i s}}=\alpha+f_{\text {tag }}\left(\right.$ month $\left._{s}\right)+\varepsilon_{i s}$ & 11 & -590.62 & 1203.23 & 121.75 & 0.000 & 0.00 \\
\hline
\end{tabular}

$D P_{1000}$ is the probability of detecting an acoustic transmission at 1000-m transmitter-receiver spacing. Subscript $i$ represents individual transmitters ( $n=8$ ), and subscript $s$ is the unit of time. Each line represented a geographic location in Lake Huron and included 2 acoustic transmitters (tag) located approximately $250 \mathrm{~m}$ apart. Residual error $(\varepsilon)$ represents residual error unaccounted by model. The variable month was represented numerically as the month of the observation during the experimental trial. All models included an AR1 autocorrelation structure to account for autocorrelation structure in data. Model degrees of freedom ( $d f$ ), log likelihood (LL), Akaike information criterion (AIC), delta AIC, Akaike weight, and estimated coefficient of determination $\left(R^{2}\right)$ are summarized for each candidate model

for all transmitters and lines indicated the relationship between $D P_{1000}$ and month was similar within lines, but differed among lines. Inclusion of a 'transmitter' term in the most parsimonious model explained more variation in the dataset than candidate models with a 'line' or an intercept term (grand mean) and was consistent 
with differences in average detection probability among acoustic transmitters.

Nonlinear trends were observed in all additive model smoothers derived from the most parsimonious model for the month-transmitter detection probability $\left(D P_{1000}\right)$ relationship for all lines (Table 3). At line 1, predicted $D P_{1000}$ was lowest in January and December, increased during the spring, and was highest during May-June (Fig. 3). In contrast, $D P_{1000}$ at lines 2 and 4 were relatively constant during the spring, fall, and winter but decreased during summer (Fig. 3). Line 3 had a similar pattern as observed at lines 2 and 4 , but the magnitude of difference among months was less (Fig. 3). Predicted $D P_{1000}$ ranged from approximately 0.6 to 0.9 at line 1 and 0.2 to 0.7 at lines $2-4$ (Fig. 3). Mean $\mathrm{DP}_{1000}$ using the most parsimonious model ranged from 0.35 to 0.8 (Fig. 4) for each acoustic transmitter and was substantially higher for transmitter at line 1 than all other transmitters (Fig. 4). Mean predicted $D P_{1000}$ differed significantly among lines but did not differ within lines except for acoustic transmitters 3 and 4 in line 2 (Table 4).

The simulated probability of detecting a fish moving through a line of receivers $D P_{\text {pass }}$ varied as a function of range detection curve used (i.e., transmitter), receiver spacing, and movement rates of virtual fish (Fig. 5). In all simulated scenarios, decreases in $D P_{\text {pass }}$ consisted of discrete short-duration (i.e., days) events and did not mirror seasonal and multi-year patterns observed in $D P_{1000}$ from Lake Huron. As movement rate of simulated fish increased, $D P_{\text {pass }}$ decreased regardless of inter-receiver spacing; however, increasing inter-receiver spacing and differences in range detection curves among stations were dominant factors that influenced $D P_{\text {pass }}$. All virtual fish moving at 0.5 or $1.0 \mathrm{~m} \mathrm{~s}^{-1}$ were detected on

Table 3 Model output of nonlinear component of most parsimonious generalized additive model (selected from among nine candidate models, see Table 2) to describe monthly patterns in 1000-m transmitter detection probability at 4 receiver lines in Lake Huron

\begin{tabular}{llll}
\hline Smooth term & edf & $\boldsymbol{F}$ & $\boldsymbol{p}$ \\
\hline Line 1 & 4.952 & 4.973 & 0.000 \\
Line 2 & 3.912 & 1.843 & 0.001 \\
Line 3 & 3.550 & 0.702 & 0.084 \\
Line 4 & 4.072 & 1.294 & 0.007 \\
\hline
\end{tabular}

A smoothed term for each receiver line was included in the most parsimonious model. Estimated degrees of freedom (edf) represent the degree of nonlinear patterns in the smoother line (linear relationship $=1$, edf values $>1$ indicate increasing nonlinear structure). $F$ and $p$ represent summary of statistical tests for a relationship between months and the probability of detecting an acoustic transmitter for each smoothing function (i.e., test of null hypothesis $=0$ for each smoothing function). Autocorrelation structure was included to account for patterns in model residuals. See Fig. 1 for map of study area all simulated days with 500-m inter-receiver spacing for acoustic transmitter 1 (Table 5). Acoustic transmitter detection probability on a virtual line $\left(D P_{\text {pass }}\right)$ with receivers spaced $500 \mathrm{~m}$ apart for transmitters 3,5 , and 7 was slightly lower than observed for transmitter 1 . For transmitters 3,5 , and 7 , all fish were detected on more than $90 \%$ of simulated days at both simulated movement rates and a virtual line of receivers spaced $500 \mathrm{~m}$ apart (Table 5). More than $85 \%$ of days modeled had estimated $D P_{\text {pass }}$ of 1.0 at $1000-\mathrm{m}$ inter-receiver spacing with 0.5 or $1.0 \mathrm{~m} \mathrm{~s}^{-1}$ movement rates (Table 5). The lowest percentage of days in which all simulated fish were detected occurred at 3000-m inter-receiver spacing and ranged from approximately $43 \%$ at transmitter 3 to $95 \%$ at transmitter 1 (Fig. 5, Table 5). Simulated $D P_{\text {pass }}$ at $1000 \mathrm{~m}$ was higher for all fish movement rates compared to transmitter detection probability $\left(D P_{\text {pass }}\right)$ estimated for the same receiver line in this study.

\section{Discussion}

Our study was unique in that we documented seasonal changes in acoustic transmitter detection probability for nearly 2 years at four separate locations in Lake Huron. Detection probability differed among months, but this variation was inconsistent through time and among receiver lines. Therefore, results from short-term range detection studies (e.g., few weeks or months) would not have been representative of transmitter detection probabilities over longer time intervals of at least 1 year. In systems like Lake Huron, deployment of range testing transmitters at several locations and for the duration of a study may be necessary to identify locations and periods of time (at monthly temporal scales) when detection probability was low enough for fish to have passed without detection. Observed fluctuations in acoustic transmitter detection probability are likely system specific and related to fluctuating environmental conditions (e.g., waves, ice, vegetation, and precipitation) between transmitter and receiver at the time of the acoustic transmission, although the time resolution at which detection probability is calculated may influence the magnitude of fluctuations [17, 19, 36, 37].

Detection of an acoustic transmission is a complex process that depends on numerous variables operating at regional and local scales [19]. Although our patterns in acoustic transmitter detection probability did not differ for transmitters deployed within $250 \mathrm{~m}$ of each other, predicted mean $D P_{1000}$ calculated for the entire time series differed for paired acoustic transmitters at line 2. Differences in mean detection probability of transmitters located within $250 \mathrm{~m}$ of each other likely reflect local processes operating at small spatial scales such as patchy plankton blooms, emergent vegetation, 


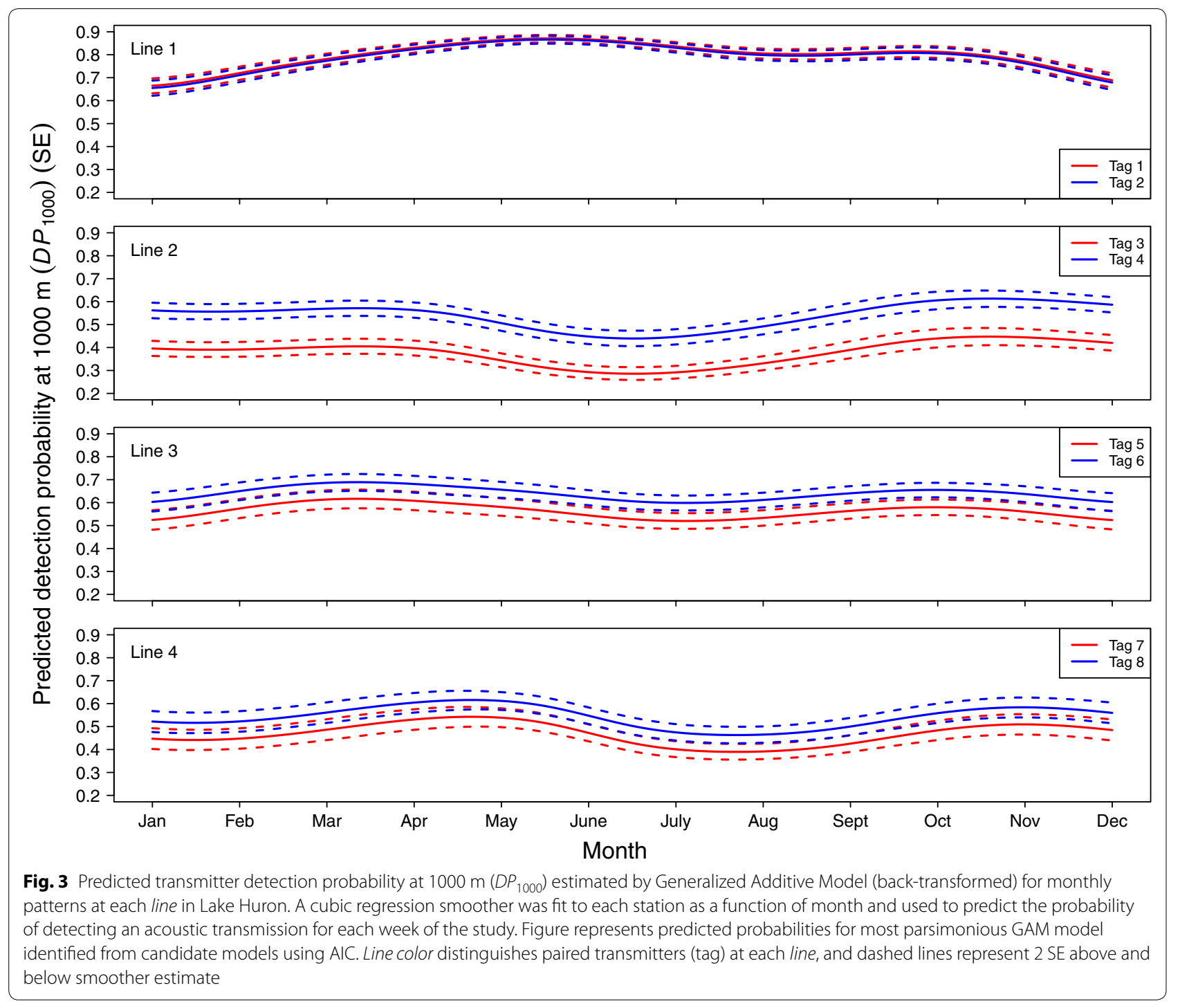

ambient noise, or the specific configuration of the physical environment, especially the presence of hard, rocky substrate that results in echoes, or variation among configurations of mooring systems [7, 37, 38]. Weather conditions or other environmental conditions acting at a regional scale were unlikely to have differentially influenced detection of multiple acoustic transmitters deployed in close proximity. The environmental causes for the variation in transmitter detection probability were not obvious in this study despite initial attempts to link transmitter detection probability with fluctuations in wind, waves, and precipitation. The inability to link transmitter detection probability with environmental conditions was likely the result of complex interactions among multiple environmental and biotic variables. Fluctuations in environmental characteristics were also not measured at appropriate spatial and temporal resolution to link fluctuations in transmitter detection probability with environmental and abiotic conditions. Other sources of noise that may influence detection probability include noise from operating boats in the vicinity of the receivers and noise associated with grinding ice during winter or the angle between receiver and transmitter. Although we did not quantify boat traffic near our receivers, intermittent noise associated with boats passing near the receivers was unlikely to have impacted transmitter detection probability calculated at weekly time intervals. Likewise, we did not quantify noise associated with grinding ice flows near our receivers. Seasonal trends observed in acoustic transmitter detection probability in this study may be partially explained by ice formation and break-up in Lake Huron. We assumed 


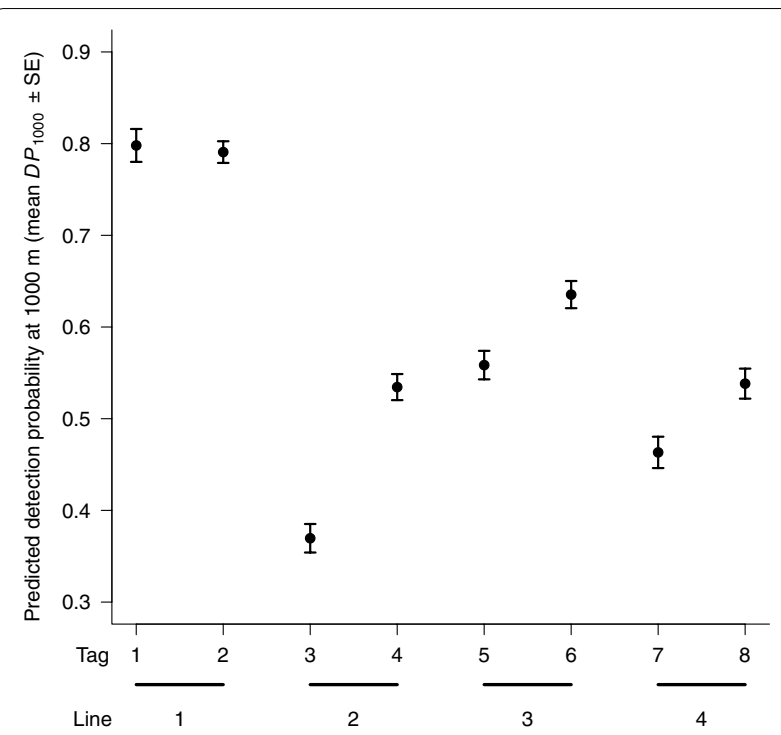

Fig. 4 Back-transformed mean predicted probability of detecting an acoustic transmission estimated from generalized additive model at $1000 \mathrm{~m}\left(D P_{1000}\right)$ transmitter-receiver distance in Lake Huron. Predicted probability was estimated for 8 transmitters (tag). Error bars represent back-transformed $95 \%$ confidence intervals

that the fluctuations in the angle between receiver and transmitter owing to the design of our transmitter and receiver mooring did not influence detection probability. Observations of acoustic receivers by SCUBA divers at locations throughout the Great Lakes suggest receivers remain nearly motionless and within a few degrees of vertical when surface conditions are safe for diving. It is unknown how receivers reacted when surface conditions were unsafe for diving.

$D P_{\text {pass }}$ varied substantially at daily time intervals in simulations with $3000-\mathrm{m}$ receiver spacing and for all simulations with empirical detection range curves from line 3. Simulated $D P_{\text {pass }}$ incorporated daily range detection curves, and observed variability in $D P_{\text {pass }}$ strongly depends on the shape of the detection range curve. Therefore, the suite of abiotic and biotic variables at sub-day temporal scales (i.e., patchy algal blooms, wind, waves, and ice dynamics) that resulted in variable daily detection range curves also impacted $D P_{\text {pass }}$. Furthermore, it is not surprising that day-to-day variability in $D P_{\text {pass }}$ was highest for simulations at $3000-\mathrm{m}$ inter-receiver distances because of the strong negative relationship between detection probability and distance between transmitter and receiver.

The probability of detecting an acoustic transmission on a single receiver within a line is often used as an index of transmitter detection probability of a line, likely because static range testing procedures are easier to conduct over multiple-day time periods or inclement weather conditions compared to mobile range testing [23]. If receiver spacing, acoustic transmission scheme, and fish movement rate provide multiple opportunities for transmitting and detecting a transmitter while the fish is moving through a receiver line, the probability of detecting the transmitter on each receiver may be low but can result in high acoustic transmitter detection probability for the line because only a single detection is necessary to confirm the presence of a fish [23]. In contrast, high probability of detecting a non-moving transmitter on an individual receiver may correspond to low transmitter detection rates on a line of multiple receivers if fish movement rates are high and the time interval between acoustic transmissions is long with the result of a fish moving through a line without transmitting an acoustic signal [23]. Few studies consider the relationship between acoustic transmitter detection probability of receivers and lines when assessing receiver line performance. The probability of detecting an acoustic transmission may not accurately reflect the probability of detecting a fish moving through a line of receivers, so simulations like the one

Table 4 Simultaneous pairwise comparisons of mean detection probability for acoustic transmitters (tag) deployed at eight locations within four lines in Lake Huron (see Fig. 1)

\begin{tabular}{llllrrrr}
\hline Tag & $\mathbf{1}$ & $\mathbf{3}$ & $\mathbf{4}$ & $\mathbf{5}$ & $\mathbf{6}$ & $\mathbf{7}$ \\
\hline 1 & 1.000 & $<0.001$ & $<0.001$ & $<0.001$ & $<0.001$ & $<$ \\
2 & & $<0.001$ & $<0.001$ & $<0.001$ & $<0.001$ & $<0.001$ & $<1$ \\
3 & & $<0.001$ & $<0.001$ & $<0.001$ & 0.061 & $<0.001$ \\
4 & & & 0.995 & 0.024 & 0.357 & 1.000 \\
5 & & & & 0.219 & 0.097 & 0.999 \\
6 & & & & & 0.001 & 0.999 \\
7 & & & & & & 0.356 \\
8 & & & & & & \\
\hline
\end{tabular}

Values represent the probability of $\mu_{a}-\mu_{b}=0$, where $\mu_{a}$ is the mean detection probability for any transmitter and $\mu_{b}$ is the mean detection probability for any other transmitter. Comparisons of paired transmitters within a line are italicized 


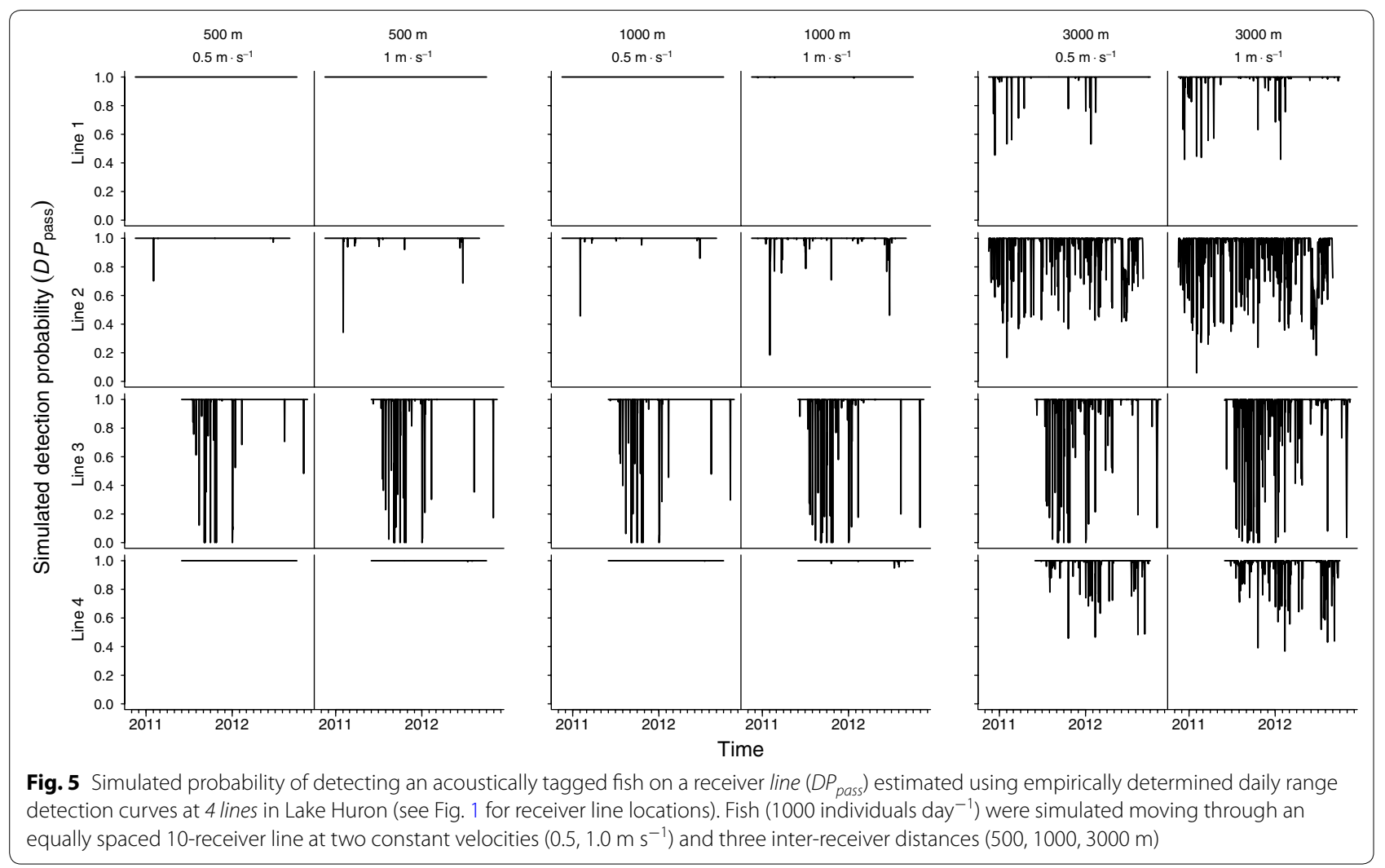

Table 5 Percent of days with line detection probability $\left(D P_{\text {pass }}\right)>0.80$ or $\left(D P_{\text {pass }}\right)$ equal to 1.0 estimated by simulating virtual fish moving through a receiver line

\begin{tabular}{|c|c|c|c|c|c|c|}
\hline \multirow[t]{2}{*}{ Tag } & \multirow[t]{2}{*}{ Line } & \multirow{2}{*}{$\begin{array}{l}\text { Receiver } \\
\text { spacing }\end{array}$} & \multicolumn{2}{|c|}{ Days $\geq 0.80$} & \multicolumn{2}{|c|}{ Days $=1.0$} \\
\hline & & & $0.5 \mathrm{~m} \mathrm{~s}^{-1}$ & $1.0 \mathrm{~m} \mathrm{~s}^{-1}$ & $0.5 \mathrm{~m} \mathrm{~s}^{-1}$ & $1.0 \mathrm{~m} \mathrm{~s}^{-1}$ \\
\hline 1 & 1 & 500 & 100.0 & 100.0 & 100.0 & 100.0 \\
\hline 3 & 2 & 500 & 99.8 & 99.7 & 99.0 & 96.2 \\
\hline 5 & 3 & 500 & 96.4 & 95.1 & 94.0 & 90.6 \\
\hline 7 & 4 & 500 & 100.0 & 100.0 & 100.0 & 99.0 \\
\hline 1 & 1 & 1000 & 100.0 & 100.0 & 100.0 & 99.3 \\
\hline 3 & 2 & 1000 & 99.8 & 98.8 & 96.6 & 87.5 \\
\hline 5 & 3 & 1000 & 95.9 & 94.0 & 92.3 & 86.7 \\
\hline 7 & 4 & 1000 & 100.0 & 100.0 & 99.4 & 96.7 \\
\hline 1 & 1 & 3000 & 98.4 & 98.1 & 95.0 & 86.7 \\
\hline 3 & 2 & 3000 & 85.0 & 79.5 & 61.2 & 43.1 \\
\hline 5 & 3 & 3000 & 92.3 & 88.4 & 78.5 & 66.5 \\
\hline 7 & 4 & 3000 & 96.3 & 93.7 & 80.2 & 62.6 \\
\hline
\end{tabular}

Simulation results included three distances $(500,1000$, and $3000 \mathrm{~m})$ and two movement rates $\left(0.5,1.0 \mathrm{~m} \mathrm{~s}^{-1}\right)$. Virtual fish $(N=1000 /$ day $)$ were swum through virtual receiver line, and detection of acoustic transmissions was simulated using daily range detection curves at four locations in Lake Huron

described here are needed to evaluate receiver line performance in the context of fish movement, rather than acoustic transmissions.

Identifying a model that accurately represents detection range curves is a primary challenge in detection range analysis because appropriate curves may differ among aquatic systems, locations within systems, and time of year. Detection range curves have been described using various linear and nonlinear functions based on empirical observations or the physics of sound propagation in 
an aquatic environment [19]. A comparison of several linear and nonlinear models to describe detection range revealed that a sigmoidal model best modeled detection range in a marine system using transmitters ranging from 136 to $158 \mathrm{~dB}[7,36]$. Although the best model for representing detection range was not explicitly tested in this study, the majority of pseudo- $R^{2}$ (i.e., goodness of fit) values for logistic models used in this study were higher than 0.8 , suggesting that the logistic function was reasonable for modeling detection range [36]. However, the logistic function did not accommodate decreased detection probability at receivers $(N=1$ for each transmitter) when receivers were located less than $400 \mathrm{~m}$ from transmitters, as was observed during several time intervals in this study. This result may seem odd, considering that acoustic transmissions were often detected well beyond $1 \mathrm{~km}$. Non-detection of acoustic transmissions at close range was consistent with close proximity detection interference caused by echoes originating from acoustic transmissions, preventing successful decoding [39]. This phenomenon is linked to the composition of substrate (e.g., mud, rock, and sand); presence of other reflective materials; orientation of the substrate, acoustic transmitter, and receiver to each other; and the power of the acoustic transmission. Close proximity detection interference is limited to transmitter-to-receiver distances of less than $400 \mathrm{~m}$ [39]. The experimental design of this study resulted in a maximum of one receiver within $400 \mathrm{~m}$ of each transmitter; logistic regression coefficients were similar for detection range curves modeled with and without receivers less than or equal to $400 \mathrm{~m}$ from the transmitter. Therefore, it is unlikely that close proximity detection interference (as per [39]) strongly influenced detection range curves in this study.

We used a two-stage analysis that combined logistic regression to estimate detection range curves and GAMs to characterize seasonal patterns in $D P_{1000}$. Alternatively, we could have used a mixed-effect binomial GAM model to characterize seasonal patterns in $D P_{1000}$. This approach would have allowed us to account for variance in the detection probability-distance within weeks. However, an attempt to fit a mixed-effect binomial GAM using the 'mgcv' package (version 1.8-12) to our data failed to converge on a result. This is not surprising because the 'GAMM' function in 'mgcv' is known to perform poorly with binary data [40]. Modeling the detection probability-distance relationship separately and subsequent analyses of transformed estimates of $D P_{1000}$ with GAMs allowed us to characterize the variability in the probability of detecting an acoustic transmission at $1000 \mathrm{~m}$ at the expense of including within-week variability in detection probability. Not including this source of variability in GAMs could bias results. However, inspection of logistic model results and goodness-of-fit metrics suggests that unexplained variation in weekly distance-detection probability relationship was minimal and unlikely influenced the results of the GAM. Furthermore, our analysis allowed us to easily incorporate standard alternative error structures to accommodate time autocorrelation in $D P_{1000}$.

This study assumed that transmitter collisions, or the simultaneous detection of multiple acoustic transmissions that results in undecipherable transmitter codes, did not occur. The frequency of transmitter collisions is dependent on the number of transmitters detected simultaneously on the same receivers, the duration of each coded signal, and average duration of time between acoustic transmissions [18]. Paired transmitters at each line were detected on the same receivers, and if transmissions occurred at the same time, these transmissions could have resulted in transmitter collisions. However, simulations of transmitter collisions for increasing numbers of transmitters simultaneously transmitting (average delay $=600 \mathrm{~s}$ ) suggested that transmitter collisions were unlikely when fewer than four transmitters were present in the system (Vemco online collision calculator, http://vemco.com/collision-calculator/, accessed June 25, 2015). In addition to transmitter collisions resulting from paired transmitters, walleye tagged with acoustic transmitters were released in Lake Huron and Saginaw Bay in spring 2011 and could have caused transmitter collisions if the fish were in proximity of the same receivers that detected stationary transmitters [24]. During our study period, the maximum number of tagged walleye detected on any receiver within the same hour was 12 . Using the same transmitter collision calculator, 12 fish transmitters simultaneously transmitting with an average delay of $120 \mathrm{~s}$ simultaneously could result in a $38 \%$ reduction in detections. Therefore, actual detection probabilities during our study could have been up to $38 \%$ higher than estimated, but such occurrences were rare. Inspection of detection probability time series before and after release of tagged fish in Lake Huron and Saginaw Bay also did not reveal any indication of excessive transmitter collisions caused by tagged walleye passing by our stationary acoustic transmitters during the study. However, future range testing studies should be cognizant of potential effects of code collisions on detection range estimates and should attempt to separately account for effects of collisions and other processes that affect signal attenuation and interference.

\section{Conclusions}

Researchers should consider the effects of fluctuating transmitter detection range on interpretation and design of telemetry studies at temporal and spatial scales 
consistent with their study objectives. Short-duration preliminary range testing at limited spatial and temporal scales may not capture variation in acoustic transmitter detection probability observed at large spatiotemporal scales. The irregular variation in transmitter detection probabilities we observed among receivers in Lake Huron makes designing receiver lines in similar systems challenging and emphasizes the need for retrospective analysis of acoustic transmitter detection probabilities. Although transmitter detection probability of individual receivers in a line may fluctuate at seasonal timescales, incorporation of multiple receivers in a line can serve to buffer these fluctuations and improve the probability of detecting a transmitter. Based on the results of our simulations, in waters ranging from $10-30 \mathrm{~m}$ in depth in Lake Huron with $69 \mathrm{kHz}$ transmitters operating at $158 \mathrm{~dB}$, receivers placed $1000 \mathrm{~m}$ apart will ensure a $90 \%$ probability of detecting a tagged fish on a receiver line over a 2-year period. Planning for, and inclusion of, static range testing using stationary transmitters as a part of a study design facilitates retrospective analysis of detection probability over the full range of environmental conditions during a study and allows the researcher to identify periods of time when performance of the acoustic transmitter-receiver system may have been poor. Combining static range testing with simulation modeling of line detection probability provides a framework for researchers to optimize telemetry system design prior to releasing fish and retrospectively evaluate the performance of existing receiver lines.

\section{Additional file}

Additional file 1. Supplement 1: R script for simulating acoustic transmitter detection probability of fish moving through a line of acoustic receivers.

\section{Abbreviations}

GAM: generalized additive model; $D P_{1000}$ : probability of detecting an acoustic transmission at $1000 \mathrm{~m}$; $D P_{\text {pass: }}$ : probability of detecting a passing acoustic transmitter moving at a constant rate on one or more acoustic receivers.

\section{Authors' contributions}

TAH led aspects of study design, executed the field portion of the study, conducted data analyses, and drafted the manuscript. CMH, TRB, JMD, SJC, CSV, and CCK participated in the design of the study, provided consultation on statistical analyses, and helped to draft the manuscript. All authors read and approved the final manuscript.

\footnotetext{
Author details

${ }^{1}$ Center for Systems Integration and Sustainability, Department of Fisheries and Wildlife, Michigan State University, Hammond Bay Biological Station, 11188 Ray Road, Millersburg, MI 49759, USA. ${ }^{2}$ U.S. Geological Survey, Great Lakes Science Center, Hammond Bay Biological Station, 11188 Ray Road, Millersburg, MI 49759, USA. ${ }^{3}$ Great Lakes Fishery Commission, 2100 Commonwealth Blvd. Ste. 100, Ann Arbor, MI 48105, USA. ${ }^{4}$ Fish Ecology and Conservation Physiology Laboratory, Department of Biology, Carleton University, 1125 Colonel By Drive, Ottawa, ON K1S 5B6, Canada. ${ }^{5}$ Ohio Department of Natural
}

Resources, Division of Wildlife, Sandusky Fisheries Research Station, 305 East Shoreline Drive, Sandusky, OH 44839, USA. ${ }^{6}$ Center for Systems Integration and Sustainability, Department of Fisheries and Wildlife, Michigan State University, 1405 South Harrison Road1 15 Manly Miles Building, East Lansing, MI 48823-5243, USA.

\section{Acknowledgements}

We thank the U.S. Geological Survey for logistical and technical support. Thanks go to Karen Murchie for her help in designing this study. This work was funded by the Great Lakes Fishery Commission by way of Great Lakes Restoration Initiative appropriations (GL-00E23010-3). This paper is contribution 21 of the Great Lakes Acoustic Telemetry Observation System (GLATOS). Any use of trade, firm, or product names is for descriptive purposes only and does not imply endorsement by the U.S. Government.

\section{Competing interests}

The authors declare that they have no competing interests.

\section{Availability of data and materials}

The data supporting the results of this article are stored in the Great Lakes Acoustic Telemetry Observation System (GLATOS) database (http://data.glos. us/glatos). Data availability is subject to data sharing policies currently under development by GLATOS, the Great Lakes Fishery Commission, and the Unites States Geological Survey.

\section{Ethics approval}

All applicable international, national, and/or institutional guidelines for the care and use of animals were followed.

\section{Funding}

This work was funded by the Great Lakes Fishery Commission by way of Great Lakes Restoration Initiative appropriations (GL-00E23010-3).

Received: 4 February 2016 Accepted: 17 October 2016

Published online: 08 November 2016

\section{References}

1. Swingland IR, Greenwood PJ. The ecology of animal movement. Oxford: Clarendon Press; 1983.

2. Nathan R, Getz WM, Revilla E, Holyoak M, Kadmon R, Saltz D, et al. A movement ecology paradigm for unifying organismal movement research. Proc Natl Acad Sci. 2008;105:19052-9.

3. Lucas MC, Baras E. Migration of freshwater fishes. Oxford: Blackwell Science; 2001.

4. Secor DH. Migration ecology of marine fishes. Baltimore: Johns Hopkins University Press; 2015.

5. Hussey NE, Kessel ST, Aarestrup K, Cooke SJ, Cowley PD, Fisk AT, et al. Aquatic animal telemetry: a panoramic window into the underwater world. Science. 2015;348:1255642.

6. Donaldson MR, Hinch SG, Suski CD, Fisk AT, Heupel MR, Cooke SJ. Making connections in aquatic ecosystems with acoustic telemetry monitoring. Front Ecol Environ. 2014;12:565-73.

7. Heupel MR, Semmens JM, Hobday AJ. Automated acoustic tracking of aquatic animals: scales, design and deployment of listening station arrays. Mar Freshw Res. 2006:57:1-13.

8. Cooke SJ, Midwood JD, Thiem JD, Klimley P, Lucas MC, Thorstad EB, et al. Tracking animals in freshwater with electronic tags: past, present and future. Anim Biotelem. 2013;1:5.

9. Krause J, Krause S, Arlinghaus R, Psorakis I, Roberts S, Rutz C. Reality mining of animal social systems. Trends Ecol Evol. 2013;28:541-51.

10. Rutz C, Morrissey MB, Burns ZT, Burt J, Otis B, St Clair JJH, et al. Calibrating animal-borne proximity loggers. Methods Ecol Evol. 2015;6:656-67.

11. Medwin H. Fundamentals of acoustical oceanography. Boston: Academic Press; 1998.

12. Simpfendorfer CA, Heupel MR, Collins AB. Variation in the performance of acoustic receivers and its implication for positioning algorithms in a riverine setting. Can J Fish Aquat Sci. 2008;65:482-92. 
13. Singh L, Downey NJ, Roberts MJ, Webber DM, Smale MJ, van den Berg MA, et al. Design and calibration of an acoustic telemetry system subject to upwelling events. Afr J Mar Sci. 2009;31:355-64.

14. Payne N, Gillanders B, Webber D, Semmens J. Interpreting diel activity patterns from acoustic telemetry: the need for controls. Mar Ecol Prog Ser. 2010;419:295-301.

15. Bergé J, Capra H, Pella H, Steig T, Ovidio M, Bultel E, et al. Probability of detection and positioning error of a hydro acoustic telemetry system in a fast-flowing river: intrinsic and environmental determinants. Fish Res. 2012;125-126:1-13.

16. Gjelland $K \varnothing$, Hedger RD. Environmental influence on transmitter detection probability in biotelemetry: developing a general model of acoustic transmission. Methods Ecol Evol. 2013;4:665-74.

17. Stocks JR, Gray CA, Taylor MD. Testing the effects of near-shore environmental variables on acoustic detections: implications on telemetry array design and data interpretation. Mar Technol Soc J. 2014;48:28-35.

18. Pincock DG. False detections: What they are and how to remove them from detection data. Halifax, NS: Vemco Inc; 2012 Apr. report no.: DOC004691 Version 03. http://vemco.com/wp-content/uploads/2012/11/ false_detections.pdf.

19. Kessel ST, Cooke SJ, Heupel MR, Hussey NE, Simpfendorfer CA, Vagle $S$, et al. A review of detection range testing in aquatic passive acoustic telemetry studies. Rev Fish Biol Fish. 2014;24:199-218.

20. Pincock DG. Detection performance of lines of VR2/NR3 receivers. Halifax: Vemco Inc; 2009 June, report no.: DOC-004819 Version 01. http://vemco. com/wp-content/uploads/2012/11/line_performance.pdf.

21. Aarestrup K, Thorstad EB, Koed A, Jepsen N, Svendsen JC, Pedersen MI, et al. Survival and behaviour of European silver eel in late freshwater and early marine phase during spring migration. Fish Manag Ecol. 2008;15:435-40.

22. Balfry S, Welch DW, Atkinson J, Lill A, Vincent S. The effect of hatchery release strategy on marine migratory behaviour and apparent survival of Seymour River steelhead smolts (Oncorhynchus mykiss). PLoS ONE. 2011;6:e14779.

23. Melnychuk MC. Detection efficiency in telemetry studies: definitions and evaluation methods. In: Adams NS, Beeman JW, Eiler JH, editors. Telemetry techniques - a user guide for fisheries research. Bethesda: American Fisheries Society; 2012. p. 339-57.

24. Hayden TA, Holbrook CM, Fielder DG, Vandergoot CS, Bergstedt RA, Dettmers JM, et al. Acoustic telemetry reveals large-scale migration patterns of walleye in Lake Huron. PLoS ONE. 2014;9:e114833.
25. Kosmidis I. BRGLM: Bias reduction in binomial-response Generalized Linear Models. 2013. http://www.ucl.ac.uk/ ucakiko/software.html.

26. Firth D. Bias reduction of maximum likelihood estimates. Biometrika. 1993;80:27-38

27. Quinn GP, Keough MJ. Experimental design and data analysis for biologists. Cambridge: Cambridge University Press; 2002.

28. Domencich TA, McFadden D. Urban travel demand: a behavioral analysis. Amsterdam: North-Holland; 1975

29. Hastie T, Tibshirani R. Generalized additive models. Boca Raton: Chapman \& Hall/CRC; 1999

30. Wood SN. Generalized additive models: an introduction with R. Boca Raton: Chapman \& Hall/CRC; 2006.

31. Zuur AF, editor. Mixed effects models and extensions in ecology with R. New York: Springer; 2009

32. Burnham KP, Anderson DR. Model selection and multimodel inference: a practical information-theoretic approach. New York: Springer; 2002.

33. Hothorn T, Bretz F, Westfall P. Simultaneous inference in general parametric models. Biom J. 2008:50:346-63.

34. Seber GAF. The estimation of animal abundance and related parameters. 2nd ed. Charles Griffin; 1982 [cited 2013 Dec 20]. http://onlinelibrary. wiley.com/doi/10.1002/bimj.19740160115/abstract.

35. Peake S, McKinley RS, Scruton DA. Swimming performance of walleye (Stizostedion vitreum). Can J Zool. 2000;78:1686-90.

36. How JR, de Lestang $\mathrm{S}$. Acoustic tracking: issues affecting design, analysis and interpretation of data from movement studies. Mar Freshw Res. 2012;63:312-24.

37. Steel AE, Coates JH, Hearn AR, Klimley AP. Performance of an ultrasonic telemetry positioning system under varied environmental conditions. Anim Biotelem. 2014;2:15.

38. Clements S, Jepsen D, Karnowski M, Schreck CB. Optimization of an acoustic telemetry array for detecting transmitter-implanted fish. North Am J Fish Manag. 2005;25:429-36.

39. Kessel ST, Hussey NE, Webber DM, Gruber SH, Young JM, Smale MJ, et al. Close proximity detection interference with acoustic telemetry: the importance of considering tag power output in low ambient noise environments. Anim Biotelem. 2015 [cited 2015 Apr 2];3. http://www. animalbiotelemetry.com/content/3/1/5.

40. Wood SN. Stable and efficient multiple smoothing parameter estimation for generalized additive models. J Am Stat Assoc. 2004;99:673-86.

\section{Submit your next manuscript to BioMed Central and we will help you at every step:}

- We accept pre-submission inquiries

- Our selector tool helps you to find the most relevant journal

- We provide round the clock customer support

- Convenient online submission

- Thorough peer review

- Inclusion in PubMed and all major indexing services

- Maximum visibility for your research

Submit your manuscript at www.biomedcentral.com/submit
BioMed Central 